\title{
An uncommon peripheral nerve injury after penetrating injury of the forearm: the importance of clinical examination
}

\section{Sunderamoorthy, M Chaudhury}

Emerg Med J 2003;20:565-566

\begin{abstract}
A 22 year old woman presented to the accident and emergency department with a self inflicted stab wound to the radial side of the volar aspect of the left forearm caused by a pen knife. Her wounds were sutured on the day of injury. Over the course of next three weeks her wounds healed well but she noticed difficulty in using the hand. She therefore attended her general practitioner who suspected a possible nerve injury and referred the patient back to the A\&E department. On follow up examination, she was noticed to have a loss of finger and thumb extension and weakness of thumb abduction. Active extension of the wrist (with radial deviation) was intact. There was no sensory deficit. Posterior interosseous nerve (PIN) palsy was diagnosed and the patient was referred to the regional hand surgery unit where she underwent exploration of the wound. A complete transection of the PIN in the supinator canal was found and repaired with good functional outcome. This case reflects the importance of clinical examination in uncommon peripheral nerve injuries and appropriate referral to a specialist department in case of doubtful penetrating wound that pose a threat to an underlying important structure.
\end{abstract}

$\mathrm{P}$ enetrating injuries are commonly seen in the accident and emergency (A\&E) department and they are commonly associated with peripheral nerve injuries. It is imperative that proper examination of such wounds are carried out so as not to miss such common injuries as it can lead to drastic consequences. Traumatic posterior interosseous nerve (PIN) injury is uncommon and most occur as a complication of Moteggia fracture dislocation. ${ }^{1}$ There are very few case reports of PIN palsy resulting from laceration and penetrating injuries of the forearm. ${ }^{12}$ We report a case of this type resulting from a self inflicted knife injury to the radial side of the volar aspect of proximal forearm. The subtle clinical signs were missed on initial presentation and only detected at follow up. This case report illustrates the importance of thorough examination after apparently minor penetrating injury.

\section{CASE REPORT}

A 22 old woman presented to the A\&E department with a self inflicted stab wound to the radial side of the volar aspect of the left forearm caused by a pen knife. A $2 \mathrm{~cm}$ entry wound was present on the volar aspect of the proximal forearm with an exit wound in the dorsal aspect of the forearm. Her wounds were sutured on the day of injury.

Over the course of next three weeks her wounds healed well but she noticed difficulty in using the hand. She therefore attended her general practitioner who suspected a possible nerve injury and referred the patient back to the A\&E department.

On follow up examination, she was noticed to have a loss of finger and thumb extension and weakness of thumb abduction (fig 1). Active extension of the wrist (with radial deviation) was intact. There was no sensory deficit. PIN palsy was diagnosed and the patient was referred to the regional hand surgery unit where she underwent exploration of the wound. A complete transection of the PIN in the supinator canal was found and repaired with good functional outcome at one year.

\section{DISCUSSION}

Penetrating injuries to the forearm are common and they are frequently associated with peripheral nerve injuries. The type of nerve involved depends on the level of the penetrating injury in the forearm. The median and the ulnar nerve are commonly injured than the posterior interosseous nerve.

The deep branch of the radial nerve is often referred to as the PIN. The PIN has some unique anatomical features. ${ }^{1}$ The radial nerve divides into the superficial and deep branches at the level of the lateral epicondyle. The PIN is a pure motor nerve with the exception of several sensory branches to the wrist joint at its most terminal extent. ${ }^{3}$ The PIN enters the arcade of Frohse between the two heads of supinator muscle. At the distal end of the supinator, it divides into multiple branches. Spinner observed that the branches are arranged in two major groups. The first group (recurrent branch) supplies the superficial layer of extensor muscles (extensor digitorum communis, extensor digiti minimi, extensor carpi ulnaris) and the second group (descending branch) supplies the deep muscle layer (abductor pollicis longus, extensor pollicis longus and brevis, extensor indicis proprius)..$^{134}$

Injuries to the PIN are uncommon because of the deep course of the nerve in the forearm. Injuries to the PIN are often missed. ${ }^{2}$

Hirachi et al have categorised traumatic PIN palsy into three types:

- Type I-complete PIN palsy producing weak wrist extension with a radial drift, extension loss at the metacarpo-phalangeal joints of all the fingers and the thumb, and weak abduction of the thumb.

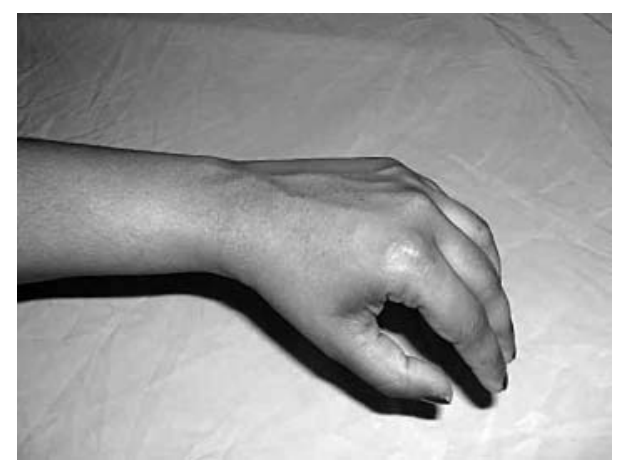

Figure 1 Loss of extension of thumb and all fingers at the MCP joint. 
- Type II-loss of extension of the little and ring fingers without loss of extension of the index, thumb and middle fingers resulting from injury to the recurrent branch.

- Type III-loss of extension of the index and the thumb and loss of abduction of the thumb without loss of extension of other fingers resulting from injury to the descending branch of the PIN.

In penetrating injuries it is imperative that the distal neurological status of the involved limb should be properly examined and recorded. One other important thing in the management of penetrating injuries is that they need to be explored if there is doubt of neurovascular damage. In the event of such doubt arising appropriate referral to a specialist department is mandatory.

In our patient with the penetrating wound the PIN palsy was missed because of incomplete examination resulting in delay in the diagnosis and subsequent management. This case therefore highlights the importance of exploring penetrating wounds and proper examination to rule out distal neurovascular deficit associated with such injuries.

\section{ACKNOWLEDGEMENT}

We wish to acknowledge our sincere thanks to Mr A Bleetman and Dr A Macnamara, consultants in accident and emergency medicine in Heartlands Hospital, for their help in preparing the article.

\section{Authors' affiliations \\ D Sunderamoorthy, M Chaudhary, Department of Accident and Emergency, Birmingham Heartlands Hospital, Birmingham, UK}

Correspondence to: Mr D Sunderamoorthy, 4 Lakeside Gardens, Merthyr Tydfil CF48 1EN, UK; dsundar6@hotmail.com

Accepted for publication 3 November 2002

\section{REFERENCES}

1 Hirachi K, Kato H, Miniami A, et al. Clinical features and management of traumatic posterior interosseous nerve palsy. J Hand Surg [Br] 1998;23:413-17.

2 Zook EG, Hurt AV, Russell RC. Sural nerve grafts for delayed repair of divided posterior interosseous nerves. J Hand Surg [Am] 1989;14A:114-20.

3 Chidges LK, Szabo RM. Radial nerve palsy-operative orthopaedics, 2nd edn. Chicago: Lippincott, 1993:1455-67.

4 Spinner M. Injuries to major branches of peripheral nerves of forearm, 2nd edn. Philadelphia: W B Saunders, 1978:80-157.

\title{
Isolated posterior gastric injury due to blunt abdominal trauma
}

\author{
A D Deshpande, S Sivapragasam
}

lising $\mathrm{n}$ civilian life most abdominal injuries are due to blunt abdominal trauma. The incidence of blunt trauma is rising because of the increase in automobile accidents. ${ }^{1}$ In blunt abdominal trauma incidence of injury to the solid organs is much higher than that to the hollow viscus.

\section{CASE REPORT}

A 17 years old man presented with a history of being knocked off a bicycle by a car and landing on his left side. He complained of pain in the left upper quadrant of the abdomen. He was haemodynamically stable and abdominal examination did not reveal anything remarkable except some bruises on the left hypochondrium and lower chest at admission but later he developed signs of peritonitis. And ultrasound scan taken at admission did not reveal any abnormality. At laparotomy the only injury found was a longitudinal tear on the posterior gastric wall in the upper third of stomach. The tear was sutured and the patient made an uneventful recovery.

\section{DISCUSSION}

Injuries to the stomach are very rare in blunt abdominal trauma. ${ }^{1}$ The stomach has very strong walls and these are not torn by blunt trauma unless it is very severe or the stomach is full, or both. Such trauma commonly involves adjacent organs like the liver, spleen, and pancreas. ${ }^{2}$ Apart from injuries to the anterior gastric wall, total rupture of the gastro-oesophageal junction, ${ }^{3}$ complete circular avulsion of the stomach from the duodenum, ${ }^{4}$ and rupture of both the gastric walls ${ }^{5}$ have been reported due to blunt abdominal trauma. All of these were associated with a solid organ injury.

In our case there was an injury only to the posterior wall of stomach. There was no other intra-abdominal injury. We, after extensive literature search, believe that this is the only case of isolated posterior gastric wall injury being reported. Because of technical problems with the computed tomography equipment an ultrasound scan was performed for this patient to exclude solid organ injury in view of the clinical signs. Mallik et al suggested that a truly normal ultrasound scan in asymptomatic patients may be highly reliable for excluding significant organ injuries and these patients may be followed up without computed tomography. ${ }^{6}$ However, in retrospect, a computed tomogram with oral contrast would probably have given an idea about the injury. The most probable mechanism of injury in this patient was injury on a full stomach.

\section{Authors' affiliations}

A D Deshpande, S Sivapragasam, Department of General Surgery, Alexandra Hospital, Redditch, UK

Correspondence to: Mr A D Deshpande, 12 Christchurch Close, Edgbaston, Birmingham B15 3NE, UK; amardd@yahoo.co.uk

Accepted for publication 6 November 2002

\section{REFERENCES}

1 Hoyt DB, Moosa AR. Abdominal injuries. In: Cuschieri A, Giles GR, Moosa AR, eds. Essential surgical practice. Oxford: Butterworth-Heinemann, 1995:531-44.

2 Wilkinson AE. Injuries of stomach. An analysis of 95 cases. S Afr J Surg 1989;27:59-60

3 Fernandez-Llamagares J, Moreno P, Garcia F, et al. Total rupture of the gastro-oesophageal junction after blunt trauma. Eur J Surg 1999;165:73-4.

4 Kolibaba SS, Shevnia PS, Khimich SD, et al. Severe closed trauma to abdomen with complete circular avulsion of stomach from duodenum and rupture of spleen and oesophagus. [In Russian]. Klin Khir 1992;4:67-8.

5 Zarowski L, Gutkowski R, Kalemba M. Rupture of both gastric walls after blunt abdominal injury. [In Polish]. Waiadomosci Lekarskie 1980:33:1255-6.

6 Mallik K, Vashisht S, Thakur S, et al. Comparative evaluation of ultrasonography and CT in patients with abdominal trauma: $A$ prospective study. Indian J Radiol Imaging 2000;10:237-43. 